\title{
Neutropenia and Thrombocytopenia Induced by Proton Pump Inhibitors: A Case Report
}

\author{
Zheng $\mathrm{Yu}^{1}$ - Jing $\mathrm{Hu}^{1} \cdot$ Yaojun $\mathrm{Hu}^{1}$
}

Published online: 23 November 2018

(c) The Author(s) 2018

\begin{abstract}
An 85-year-old man was admitted to our hospital because of dysphagia, and was diagnosed with benign stricture of the esophagus. He was hospitalized repeatedly for balloon dilations. Pantoprazole sodium ( $80 \mathrm{mg}$, twice daily, intravenously) was administered each time when he was in hospital, while esomeprazole $(20 \mathrm{mg} /$ day, orally) was administered intermittently when he was at home. Reductions in both white blood cells and platelets were noticed about 4 months after proton pump inhibitors were introduced. Bone marrow suppression induced by proton pump inhibitors was diagnosed as proven by bone marrow biopsy. White blood cell, neutrophil, and platelet counts went back to the normal range after proton pump inhibitors were stopped. The present case shows a rare bi-cytopenia associated with proton pump inhibitors and suggests the importance of awareness of hematological adverse events during proton pump inhibitor therapy.
\end{abstract}

\section{Key Points}

Proton pump inhibitors might induce leukopenia and thrombocytopenia.

Neutrophil and platelet counts may go back to the normal range after proton pump inhibitors are stopped.

Clinicians should be aware of this adverse effect even though it is very rare.

\section{Introduction}

Proton pump inhibitors (PPIs) are widely used medications for treatment of gastric acid-related diseases [1, 2]. With the increasing use of PPIs, a series of complications and adverse effects have emerged [3, 4]. Blood dyscrasias are rare adverse effects. Although some cases of cytopenia

Zheng Yu

yuzheng0928@163.com

$\triangle$ Jing $\mathrm{Hu}$

fxhujing@163.com

1 Department of Gastroenterology, Fuxing Hospital, the Eighth Clinical Medical College, Capital Medical University, \#20, Fuxingmenwai Street, Xicheng District, Beijing, China associated with PPI treatment have been reported, bi-cytopenia has not been documented [5-8]. Here, we report the first case of myelosuppression induced by PPI use, which caused both leukopenia and thrombocytopenia.

\section{Case Report}

An 85-year-old Chinese man was admitted to our hospital because of dysphagia in late June 2017. His medical history included transurethral resection of prostate for benign prostatic hyperplasia in 2012 and percutaneous vertebroplasty for lumbar vertebral compression fracture in 2015. He did not take any medicine when he was at home. The patient underwent endoscopic multi-band mucosectomy for resection of an early squamous cell carcinoma of the esophagus at 21 months previously in another hospital, and subsequently developed progressive dysphagia. $\mathrm{He}$ received four endoscopic dilations, and the dysphagia recurred soon after dilation each time. The exact results of examination and the details of treatment in the other hospital were unclear. He was able to swallow only liquids when he came to our hospital. After admission to our hospital, a physical examination revealed that he weighed $60 \mathrm{~kg}$, with a body mass index of 18.4, and had stable vital signs. No superficial lymph nodes were palpable. Abdominal examination revealed a soft, non-tender abdomen without hepatosplenomegaly. A complete blood 
count showed mild anemia with slightly reduced serum ferritin and iron concentrations (white blood cell count $5.6 \times 10^{9} / \mathrm{L}$, neutrophil count $4.46 \times 10^{9} / \mathrm{L}$, red blood cell count $2.97 \times 10^{12} / \mathrm{L}$, hemoglobin $104 \mathrm{~g} / \mathrm{L}$, platelet count $135 \times 10^{9} / \mathrm{L}$, serum iron $5.70 \mu \mathrm{mol} / \mathrm{L}$, transferin saturation $16.72 \%$, total iron binding capacity $34.10 \mu \mathrm{mol} / \mathrm{L}$ ). Iron deficiency anemia caused by malnutrition was suspected. Iron sucrose was administered intravenously and intermittently ( $100 \mathrm{mg}$, three times a week, intravenous infusion). Iron sucrose was stopped due to short hospital stay and shortage of medicine in the nursing home, with a total dose of $300 \mathrm{mg}$. An esophagoscopy and esophagogram revealed a $2-\mathrm{mm}$-long benign scar stricture. A stent was placed after dilation. Dysphagia was alleviated, and the patient was released from the hospital. The stent was dislodged from its proper location after 1 month, and dysphagia had recurred. The stent was removed and an additional balloon dilation was performed in August 2017. Dysphagia was improved markedly, but repeated half to 1 month after each dilation. The man was hospitalized later in September and December 2017 for another two dilations. Pantoprazole sodium $(80 \mathrm{mg}$, twice daily, intravenous infusion) was administered each time when he was in hospital, while esomeprazole ( $20 \mathrm{mg} /$ day, orally) was administered intermittently when he was at home. He came back to our hospital for the fourth balloon dilation on December 2017. Pantoprazole sodium was given again from hospital day 3. A relatively obvious decrease in platelets (from $135 \times 10^{9} / \mathrm{L}$, checked when he first entered our hospital in June, to $83 \times 10^{9} / \mathrm{L}$ ) was found on hospital day 5. After 4 days of pantoprazole administration, neutropenia (white blood cell count from $5.6 \times 10^{9} / \mathrm{L}$, checked when he first entered our hospital in June, to $2.67 \times 10^{9} / \mathrm{L}$; neutrophil count from $4.46 \times 10^{9} / \mathrm{L}$, checked when he first entered our hospital in June, to $0.88 \times 10^{9} / \mathrm{L}$ ) was observed on hospital day 7 . In a review of his previous medical history, we found a trend of slight decrease in white blood cells and neutrophils since his first admission to our hospital. Further examinations were performed. A bone marrow aspiration smear showed few nucleated cells, fat droplets, and scattered non-hemopoietic islands. A bone marrow biopsy indicated hypoplastic hematopoiesis. Helper T cells were in the normal range. Genetic detection of Wnt1 by reverse transcription polymerase chain reaction (RTPCR) was within the normal range. Antinuclear antibody (ANA) test was positive (1:1000, speckled pattern), while anti-dsDNA, anti-SS-A, anti-SS-B, anti-SM, anti-SCL-70, and anti-Jo-1 antibodies were all negative. Bone marrow suppression caused by PPI use was suspected due to lack of another cause. We stopped pantoprazole sodium treatment on hospital day 7 and subsequently found rebounds in white blood cell, neutrophil, and platelet counts; these values returned to normal on hospital day 15 (Fig. 1).

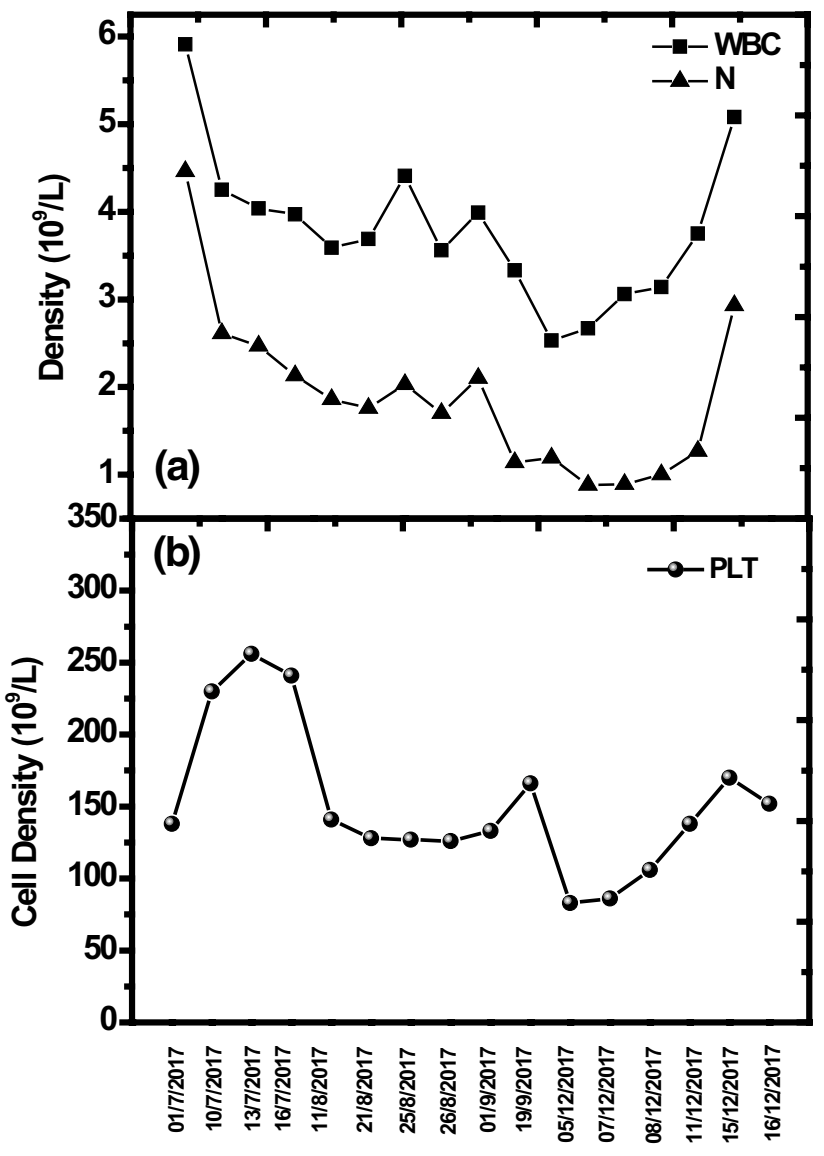

Fig. 1 White blood cell (WBC) count, neutrophil (N) count, and platelet (PTL) count were all within their normal ranges when the patient was first admitted. Proton pump inhibitor (PPI) therapy was adopted after he was hospitalized, and the levels of white blood cells and neutrophils decreased slowly afterward. The specific times when pantoprazole sodium and esomeprazole were used was as follows: pantoprazole sodium: 02/07/2017-16/07/2017, 10/08/201707/09/2017, 20/09/2017-22/09/2017, and 03/12/2017-07/12/2017; esomeprazole: 17/07/2017-09/08/2017, 08/09/2017-19/09/2017, and 23/09/2017-02/12/2017. Thrombocytopenia was found on December 5, 2017, while neutropenia appeared on December 7, 2017. PPI treatment was stopped on December 7, 2017, and the levels of these blood cells increased to normal slowly afterward

\section{Discussion}

PPIs are among the most commonly used drugs worldwide [2], and PPI-associated adverse events have emerged since use of PPIs has increased. Although the risk of neutropenia is mentioned in the package inserts of both pantoprazole and esomeprazole, case reports of neutropenia are rare. In addition to these reports regarding neutropenia, there are sporadic reports of anemia or thrombocytopenia related to PPIs [5-8]. We searched PubMed using terms including proton pump inhibitors, pantoprazole, esomeprazole, adverse effects, side effects, neutropenia, 
thrombocytopenia, cytopenia, blood dyscrasia, bone marrow suppression and myelosuppression; reports concerning adverse effects of PPI therapy on blood cells are limited to cell reduction in one of the myeloid lineages. Here, we report a case of bone marrow suppression associated with PPI exposure that induced both neutropenia and thrombocytopenia.

Blood dyscrasia is associated with many PPIs, and there may be potential drug cross-reactivities between them [5]. In the course of our patient's disease, pantoprazole and esomeprazole were used alternately. It appears that cytopenia might be attributed to either or both of them since levels of white blood cells and platelets showed a downward trend. However, neutropenia and thrombocytopenia occurred during treatment with pantoprazole and resolved soon after pantoprazole was stopped. The Naranjo Causality Algorithm was performed, and 5 points indicate a probable relationship between use of pantoprazole and cytopenia in this patient. Therefore, pantoprazole was more likely to be the cause of cytopenia in this patient.

Mechanisms of PPI-induced cytopenia are unclear. The two most commonly accepted theories are the immunemediated mechanism and toxic mechanism. The immunemediated mechanism is based on drug-induced antibodies against circulating hemocytes, whereas the toxic mechanism indicates direct toxicity of the drug to hematopoietic cells [9]. In the previous case reports, almost no bone marrow biopsies were performed. We found only one case in which bone marrow aspiration was conducted, but this report has obvious limitations because the arrest of bone marrow may be due to possible drug interactions between PPI and immunosuppressants in the renal failure patient [10]. In our report, bone marrow aspiration and biopsy were performed, which indicated suppression of bone marrow functions. Moreover, related tests were conducted to exclude other etiologies that may also lead to cytopenia. Based on the abovementioned examinations, we reached the conclusion that PPIs led to the suppression of bone marrow in this patient. In this case report, the levels of neutrophils and platelets decreased slowly after treatment with PPI began and then fell dramatically and suddenly after PPIs were used for a period of time. This result suggests that suppression of bone marrow may be aggravated by prolonged use of PPIs. In our patient, blood cells were restored to the normal range 8 days after discontinuation of PPIs. However, whether bone marrow depression is always reversible or may be irreversible as administration time is extended is still unclear.

PPIs are usually well tolerated, with very few reported side effects on hematopoietic systems. Nevertheless, it is important to be aware of this potential complication and discontinue the drug immediately when such an event occurs.

\section{Conclusion}

PPIs are among the most commonly used drugs in the treatment of a variety of diseases, including gastroesophageal reflux disease and peptic ulcer. Some patients may take these drugs for a long time, sometimes even for years, due to recurrence of diseases or symptoms. We presented a case of neutropenia and thrombocytopenia induced by PPIs in an old man after using PPIs for about 5 months intermittently. There are many causes of neutropenia and thrombocytopenia, including bacterial or viral infections, autoimmune diseases, aplastic anemia, leukemia, lymphoma and adverse effects of drugs. It is very difficult to reach the etiology of neutropenia and thrombocytopenia. Because the incidence is extremely low, PPIs as the cause of neutropenia and thrombocytopenia may be overlooked, so clinicians should be aware of this adverse effect and stop the drugs immediately when such a case is suspected.

\section{Compliance with Ethical Standards}

Funding No sources of funding were used in the preparation of this case report.

Conflict of interest Zheng $\mathrm{Yu}$, Yaojun $\mathrm{Hu}$ and Jing $\mathrm{Hu}$ declare that they have no conflict of interest.

Ethical approval Written informed consent was obtained from the patient for publication of this case report and any accompanying images.

Open Access This article is distributed under the terms of the Creative Commons Attribution-NonCommercial 4.0 International License (http://creativecommons.org/licenses/by-nc/4.0/), which permits any noncommercial use, distribution, and reproduction in any medium, provided you give appropriate credit to the original author(s) and the source, provide a link to the Creative Commons license, and indicate if changes were made.

\section{References}

1. Turner T. Proton pump inhibitors. 2018. https://www.drugwatch. com/proton-pump-inhibitors/. Accessed 10 Oct 2018.

2. Statista. Top 10 traditional therapy drugs in the U.S. by share of total traditional spending in 2016. 2017. https://www.statista. com/statistics/657151/traditional-therapeutic-drugs-in-the-usby-percent-of-total-spend/. Accessed 10 Oct 2018.

3. Freedberg DE, Kim LS, Yang YX. The risks and benefits of long-term use of proton pump inhibitors: expert review and best practice advice from the American Gastroenterological Association. Gastroentrology. 2017;152:706-15.

4. Malfertheiner P, Kandulski A, Venerito M. Proton-pump inhibitors: understanding the complications and risks. Nat Rev Gastroenterol Hepatol. 2017;14:697-710.

5. Gouraud A, Vochelle V, Descotes J, Vial T. Proton pump inhibitor-induced neutropenia: possible cross-reactivity 
between omeprazole and pantoprazole. Clin Drug Investig. 2010;30:559-63.

6. Dury S, Nardi J, Gozalo C, Lebargy F, Deslee G. Agranulocytosis induced by proton pump inhibitors. J Clin Gastroenterol. 2012;46:859.

7. Taş A. Thrombocytopenia as a side effect of pantoprazole. Turk J Gastroenterol. 2013;24:295-6.

8. Shikata T, Sasaki N, Ueda M, et al. Use of proton pump inhibitors is associated with anemia in cardiovascular outpatients. Circ J. 2015;79:193-200.
9. Heimpel H. When should the clinician suspect a drug- induced blood dyscrasia, and how should he proceed? Eur J Haematol Suppl. 1996;60:11-5.

10. Gabutti L, Stoller R, Vogt B. Omeprazole-induced agranulocytosis in a kidney transplant recipient. Nephrol Dial Transpl. 1999;14:523-4. 